Faculty of Design

2013

\title{
Teaching information visualization by exploring their power in engaging users of social media applications
}

Meirelles, Isabel and Sun, Xiaohua

Suggested citation:

Meirelles, Isabel and Sun, Xiaohua (2013) Teaching information visualization by exploring their power in engaging users of social media applications. InfoDesign - Revista Brasileira de Design da Informação, 10 (1). pp. 38-54. ISSN 1808-5377 Available at http://openresearch.ocadu.ca/id/eprint/911/

Open Research is a publicly accessible, curated repository for the preservation and dissemination of scholarly and creative output of the OCAD University community. Material in Open Research is open access and made available via the consent of the author and/or rights holder on a non-exclusive basis.

The OCAD University Library is committed to accessibility as outlined in the Ontario Human Rights Code and the Accessibility for Ontarians with Disabilities Act (AODA) and is working to improve accessibility of the Open Research Repository collection. If you require an accessible version of a repository item contact us at repository@ocadu.ca. 


\title{
Teaching information visualization by exploring their power in engaging users of social media applications
}

\author{
Isabel Meirelles, Xiaohua Sun
}

information visualization, social media applications, pedagogy
A The paper describes a novel way of introducing methods and approaches of visualizing data to undergraduate students that was conducted during a seven-week module course at the College of Design \& Innovation at Tongji University, Shanghai, China. It starts with a brief introduction to the rational and methodology used, followed by an examination of the four pedagogical phases that structured the course, including sample of student work. A discussion provides a critical view of the pros and cons of this pedagogical approach with directions for future curriculum.

\section{Introduction}

The paper describes the experience of teaching an undergraduate introductory information design course in the College of Design \& Innovation (D\&I) at Tongji University, Shanghai, China. The focus of the program at D\&I is in digital media. The class combined second and third year undergraduate students with background in motion design, user-interface design, and a solid art and design foundation, including basic principles of typography. Students did not have previous experience with programming or with visualization of information.

Similar to other introductory courses on information design, our curriculum introduced basic concepts, methods and procedures used in the visual presentation of information by means of lectures, discussions, studio projects, and critiques. Our learning objectives were that students develop basic critical language and procedures used in visual displays of information, and learn the basics of how to analyze, organize, visualize and communicate information.

Given the background of the students in digital media, the course experimented with a novel way to introduce principles of information visualization. The program of study was structured around issues that students were familiar with - social media applications - as a way to engage them into the creative process of organizing and visualizing information. For that, instead of assigning typical information design projects, that are often open-topic standalone works, we required students to apply visualization principles in the context of social media applications. We adopted the definition of visualization by Card and colleagues as 'the use of computer-supported, interactive, visual 
1 http://www.moma.org/ visit/calendar/exhibitions (Accessed April 10, 2013)

2 http://newsmap.jp (Accessed April 10, 2013) representations of abstract data to amplify cognition.' (CARD et al., 1999, 7).

The task we gave students was to devise design mechanisms to engage users of social media applications into participating in realtime interactive visualizations. In other words, we encouraged students to explore and conceive of visualizations using data generated by their own audience's interactions with selected social media services. Thus, the visualization task had a clear problemsolving goal, in which data types were imposed by the social media service of their choice. Due to the short duration of the course, the final output was a presentation that clearly demonstrated the functionalities of the proposed visualization inside the selected social media application interface.

The objective of the paper is to describe this novel way of introducing methods and approaches of visualizing data, including a critical view of the pros and cons of this pedagogical approach.

\section{Methodology}

The course was developed around two recent trends in digital applications: the use of visualization techniques as design components in interactive applications; and the wide spread adoption of social media applications by a diverse body of users.

In recent years we have seen the integration of visualization techniques as a means to both navigate digital content as well as enhance the user experience of applications (MEIRELLES, 2011). These visualizations make use of both well-known schemas and novel techniques to structure information. Take for example the interactive exhibition timeline in the website of the Museum of Modern Art in New York City ${ }^{\mathbf{1}}$ that uses the familiar encoding of the length of lines to stand for duration of events to convey information on current and future exhibits in the museum (TVERSKY, 2001). Another example is the NewsMap by Weskamp, which uses the treemap technique to visually present the hierarchy of news coverage in several countries while also providing access to the news stories ${ }^{2}$.

Social media services were selected as the platform for the project. These services have been adopted world-wide and are ubiquitous in China, where they have their own specificities and complexity. Another consideration was the level of difficulty brought by the variety of digital devices used in association with each other when interacting with social media applications. For example, in China we might search a place to eat in the computer using Dazhong Dianping system of recommendations and once at the restaurant we might use Tencent WeChat to share photos of the delicious food from our smart phone. 
3 http://www.linkedin. com/company/dianping

(Accessed April 10, 2013)
We assigned students the task to design visualizations that integrated into existing social media applications would enrich user engagement with the selected service. For that, students worked in self-selected groups and each chose a social media application to examine as follow:

- Dazhong Dianping: is an online city guide service in China. The company describes their application as "the Yelp + Groupon + Foursquare of China" ${ }^{3}$. The name roughly translates to "Reviews by/for the Masses"

- WeChat: is a mobile phone text and voice messaging communication service developed by Tencent in China. To our knowledge there has no corresponding service available in the U.S.

- Tencent QQ: is an instant messaging software service that encompasses several other applications including online social games, music, shopping, micro blogging, and group and voice chat. The platform was also developed by Tencent in China

- Instagram: is an online photo-sharing and social networking service originally developed in the U.S.

\section{Structure}

The course was structured in four main phases, covering a total of seven weeks:

- Research of the selected service (2 weeks);

- Exploration of ways to introduce visualization of data as potential new ways to engage with the social media service (2 weeks);

- Development of main visualization features (2 weeks);

- Development of a presentation of the proposed visualization, including the process by which they created their solutions (1 week);

What follows is a short description of each phase illustrated with samples of student work.

\section{Research}

The pedagogical objective of this initial phase was twofold. First to have students develop a conceptual model of the selected social media application by means of increasing their knowledge about the system while removing any pre-conceived ideas given their daily interactions with them. Second, to introduce diagrammatic techniques for structuring complex systems. The lectures in the first two weeks 
covered design methodologies, as well as provided an introduction to issues involved in the practice of visualizing data: from historical examples to current state of the field.

To accomplish these goals we introduced research methodology coupled with strategies for diagramming and creating visual representations of complex systems. Diagramming and visually presenting ideas are common techniques to all design activities (and courses). The new skills taught were principles of grouping and categorizing data, material that is usually introduced in information design courses. By combining how to group information with previous knowledge of communicating diagrammatically, students were then capable to develop the first assignment: to visually communicate the structure of the selected system. We requested students to scrutinize the selected social media applications and communicate their findings by means of a "concept map" that visually communicates the main features of the service: what it offers, how people use it, who uses it, where they use it, why they use it, and so on. Dubberly explains, 'A concept map is a picture of our understanding of something. It is a diagram illustrating how sets of concepts are related... The purpose of a concept map is to represent (on a single visual plane) a person's mental model of a concept' (DUBBERLY, 2010).

Below we reproduce the first draft created after a week of work (Figure 1), and the final concept map delivered the following week (Figure 2) by the Instagram group.1 Not only is the final map easier to understand and follow, it is also better structured. Note for example the way in which they refined the "HOW" functionalities in the second map, that separates steps in the process - from download to install - from functionalities of the service and features of the application (Figure 3).

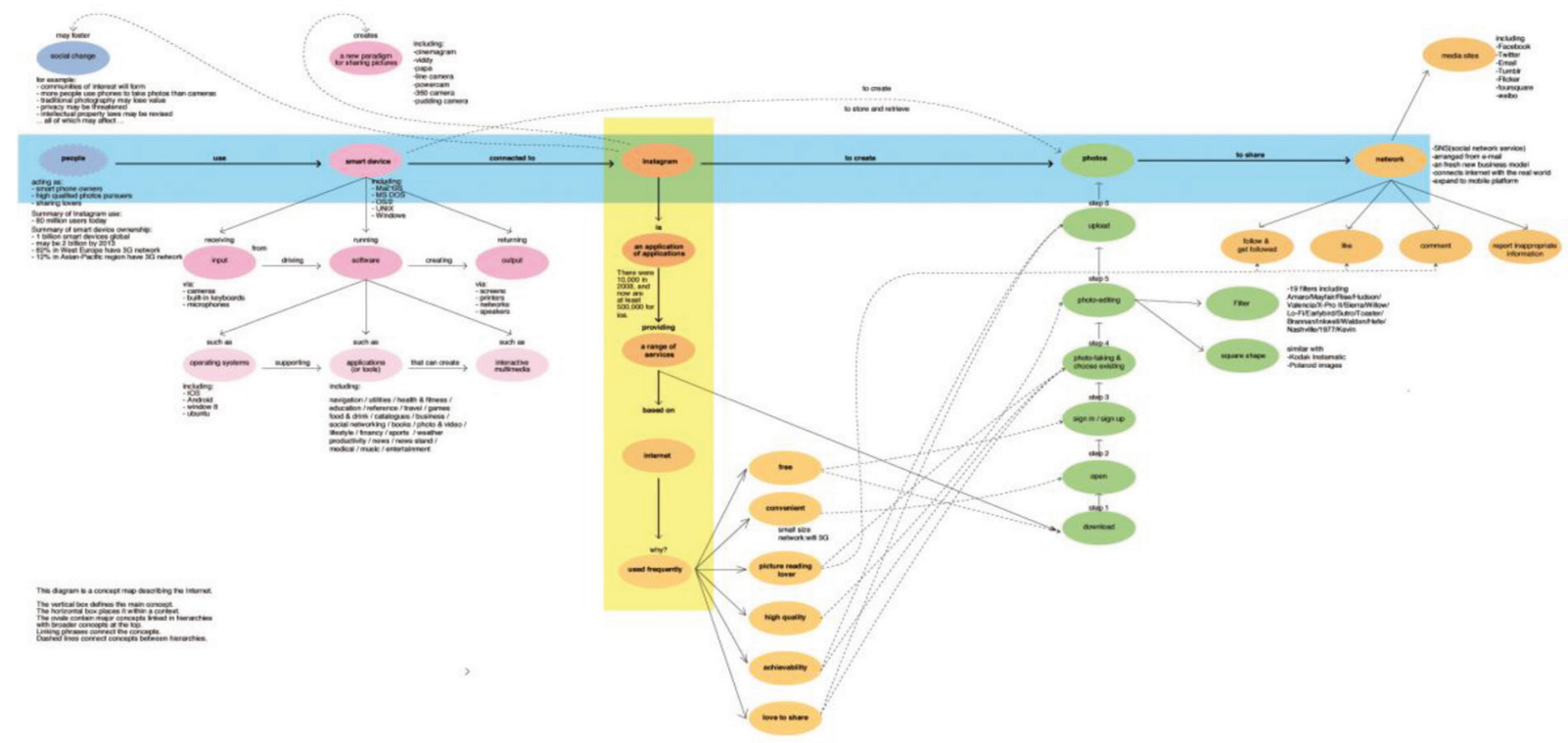

Figure 1 First draft of Instagram concept map (used with permission of students) 


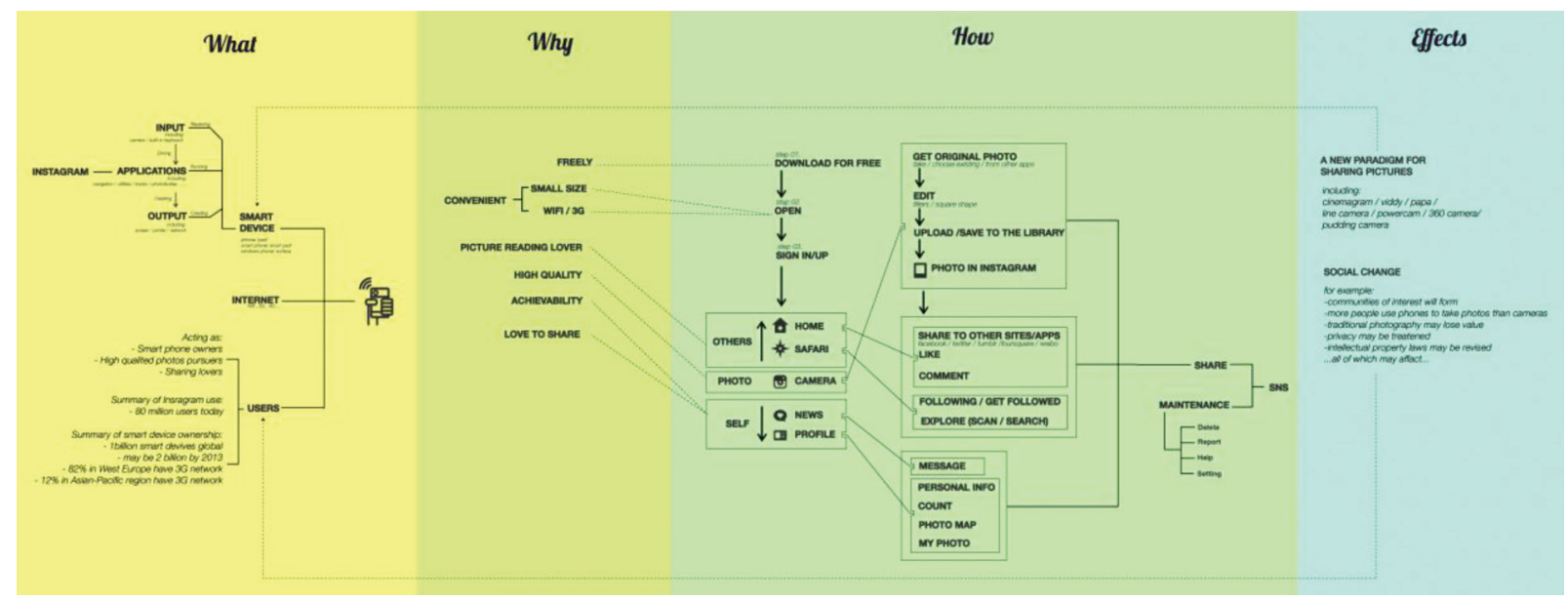

Figure 2 Final Instagram concept map (used with permission of students)
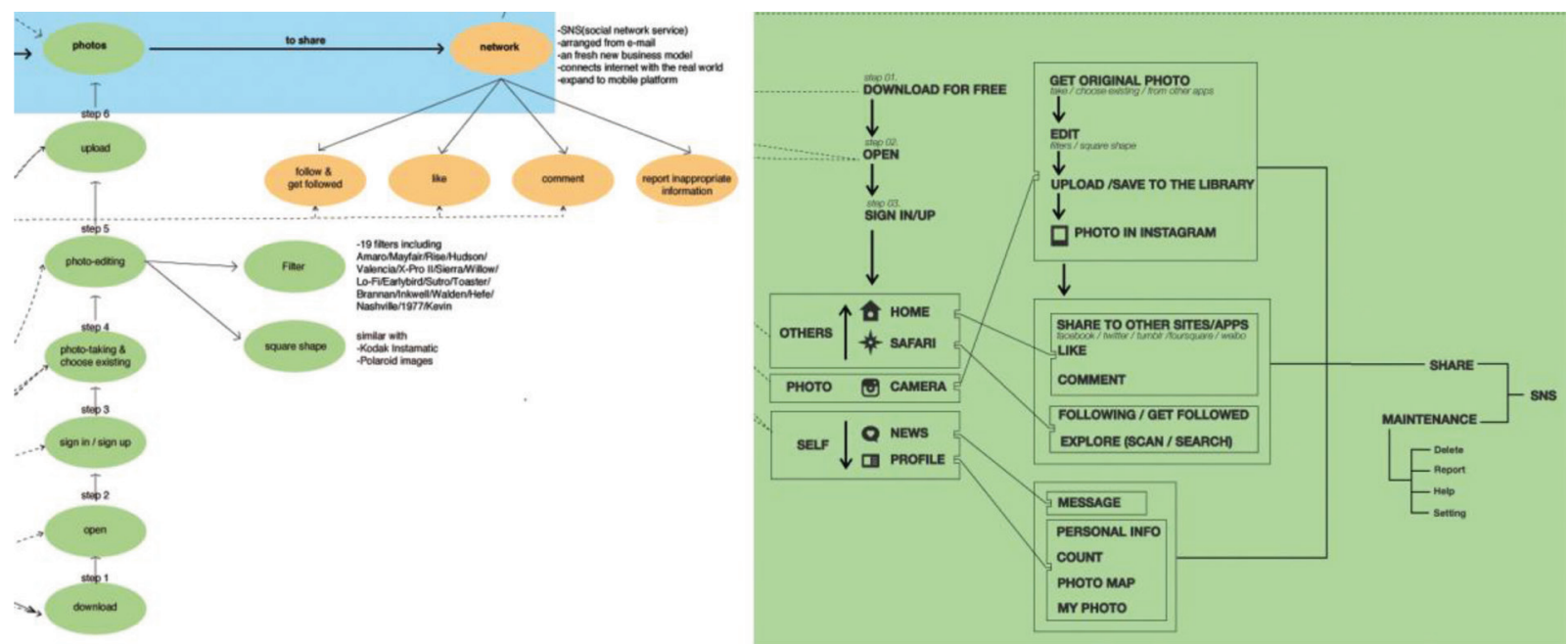

Figure 3 Comparison of the "HOW" functionalities and features between the first and second versions of the concept map (used with permission of students)

This first phase was effective to teach students basic principles of categorizing and visually structuring information in addition to basic methods of encoding data. We also noticed that students gained new insights about the applications through careful research. Similarly, students informally communicated that the research helped them learn about features they were unfamiliar with, and that they would not have known otherwise.

\section{Exploration}

The exploration phase consisted of two types of activities that ran in parallel: sketching as a problem-solving technique, and critical analysis of existent visualizations. Lectures focused on expounding the 
cognitive processes behind effective visualizations, and understanding the constraints and capabilities of visual perception in the context of information design.

The objective of this exploratory phase was to foster discovery of potential solutions by means of sketching while also expanding their knowledge of the field (analytical exercises). In other words, we encouraged students to visually express ideas by removing anxieties related to crafting "perfect' representations. Herbert A. Simon contends:

All mathematics exhibits in its conclusions only what is already implicit in its premises... Hence all mathematical derivation can be viewed simply as change in representation, making evident what was previously true but obscure. This view can be extended to all of problem solving - solving a problem simply means representing it so as to make the solution transparent... A deeper understanding of how representations are created and how they contribute to the solution of problems will become an essential component in the future of theory of design. (SIMON, 1996, 132)

Students explored potential ways to integrate interactive visualization techniques into the selected social media service through a series of sketching exercises that involved narrative strategies coupled with user scenario case studies. First, students brainstormed ideas by drawing stories based on the concept maps as well as on "dream" features. Next, each group chose a feature to storyboard for presentation and discussion. The result of the exploratory stage was then taken into the development phase described below. ideas by drawing stories based on the concept maps as well as on "dream" features. Next, each group chose a feature to storyboard for presentation and discussion. The result of the exploratory stage was then taken into the development phase described below.

For example, the Instagram group proposed to enable users of the photo social service the ability to attach a new metadata of altitude to their images, hence the name of their visualization project: "Altigram." Their concept, which is quite feasible, considered that given the geolocation of an image, the altitude would be provided by a correlated dataset of altitudes, which then would serve to rank images, such as the top 10 highest and lowest altitudes. Images could then be searched and retrieved in visualizations according to altitudes in diverse ways: in ranked lists of users, in ranked lists of altitudes, and in maps. Furthermore, the concept considered that the new visualization tools would inspire users to get outside and participate in outdoor activities for a happier and healthier life (Figure 4). 


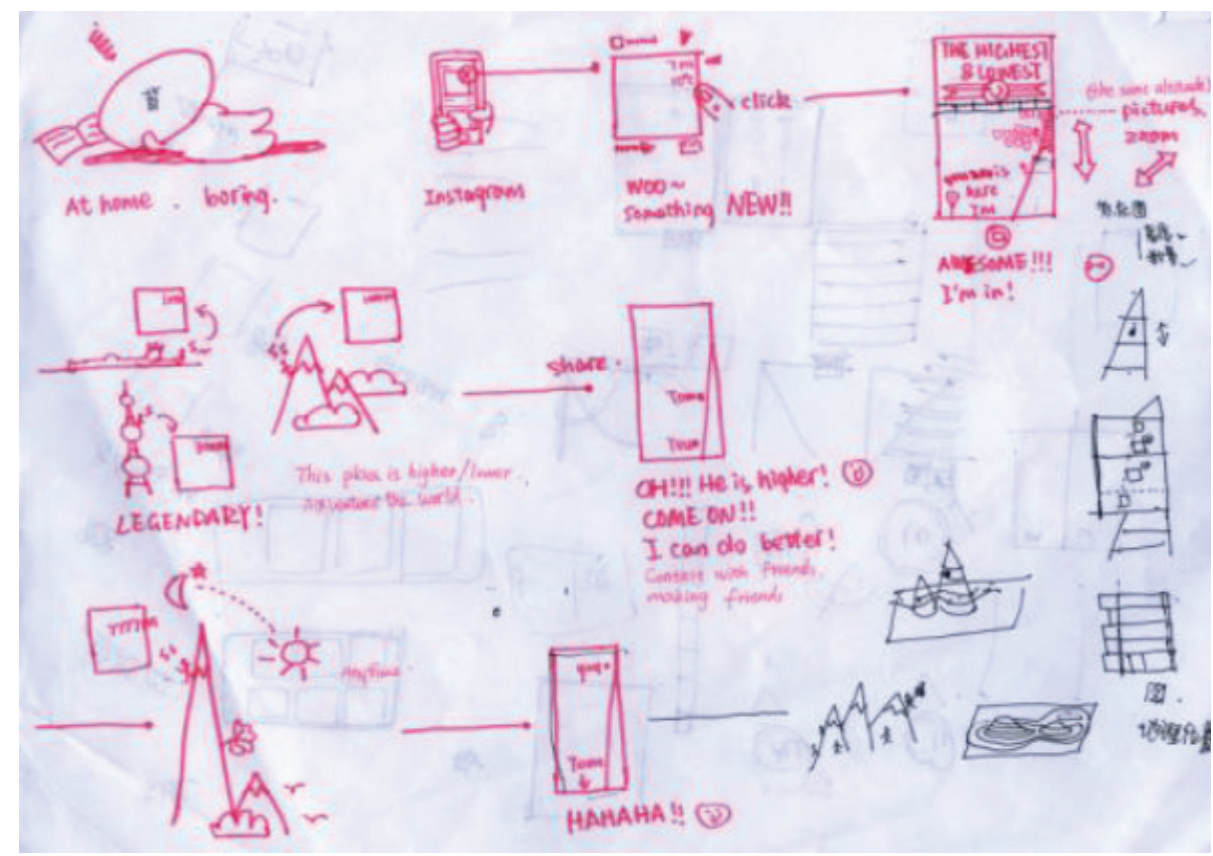

Figure 4 Storyboard of an envisioned feature for integration into the Instagram application that considers the altitude of photos and visualizes the ranked results (used with permission of students)

Considering that Instagram is a mobile device application, students designed visualizations for mobile devices, mainly smart phones. But they also considered a web application, where users could then look at maps and other visualizations structured around geographic data that would be more effective if viewed in a computer rather than on the small screen of smart phones. Below are the initial sketches that were taken to the development phase (Figure 5).

While generating ideas by means of sketching, we asked students to critically analyze existent visualizations and then present their findings to the class. Each group selected three visualizations from online repositories (e.g., visualcomplexity, flowingdata, infosthetics). The study required students to look at: the intended audience; the tasks enabled in the visualization; the types of data as well as the types of data encoding; and finally the lessons learned from the visualization (if any).

The analytical exercise had two goals. First, to ensure that the points presented in the lectures were learned and put into practice by the students. Second, to help students broaden the repertory of known visualizations and potentially become better informed while developing their own projects.

In sum, during the discovery phase students were able to keep a sense of the larger context and explore solutions to the problem at hand without being caught up in details, while also asking important questions, mostly learned from looking at projects online. The sketching exercises facilitated examination of solutions pertaining 
to different types of device, whether a smart phone or a web site (Figure 5). Furthermore, students accomplished our objective of defining the main functionality of the proposed visualization to take into the development phase.

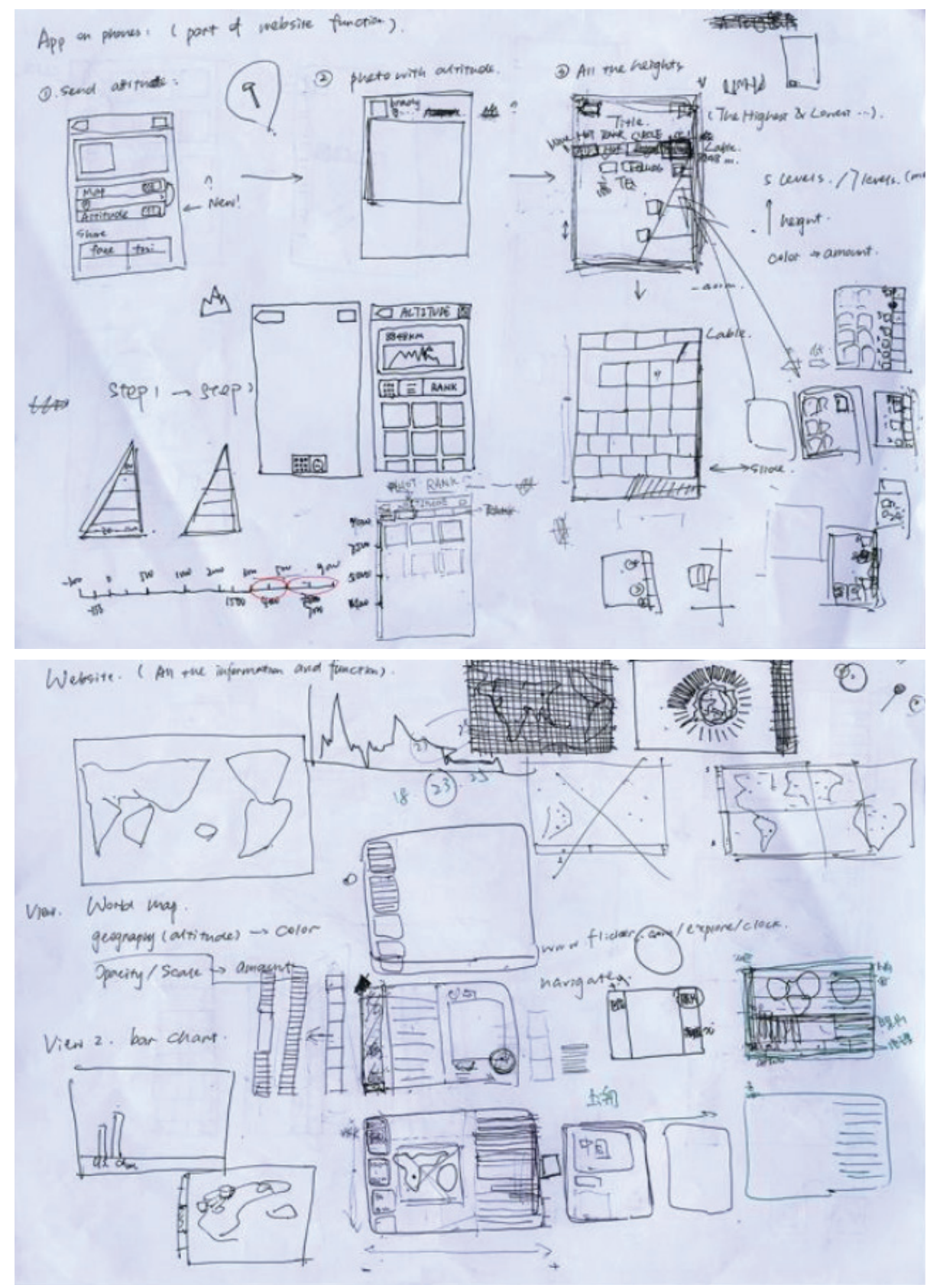

Figure 5 Initial sketches of visualization techniques for the Altigram project: for the smart phone (top), and online application (botom) (used with permission of students) 


\section{Development}

Up to this point students had conducted research about a selected social media system with the purpose, first, to understand the types of data they could use, and second, to explore and define the task of the proposed visualization. During the development phase, students were asked to translate their concepts and initial ideas into design decisions. The objective now was to foster students into making meaningful use of data structures and data encoding to afford their choices of user task. We supported their search for appropriate visualization techniques with a series of lectures focused on data types, data structures, and data encoding. In addition, we worked closely with each group to ensure that they had access to other extra material relevant to the specificities of each project.

For example, there were two groups working with the QQ application and each required a different set of skills to develop their visualizations, even though both were dealing with the same social media service. One group selected to visualize relationships between users and their music habits (Figure 6), whereas the other group focused on representing the geo-location of users over time (Figure 7).

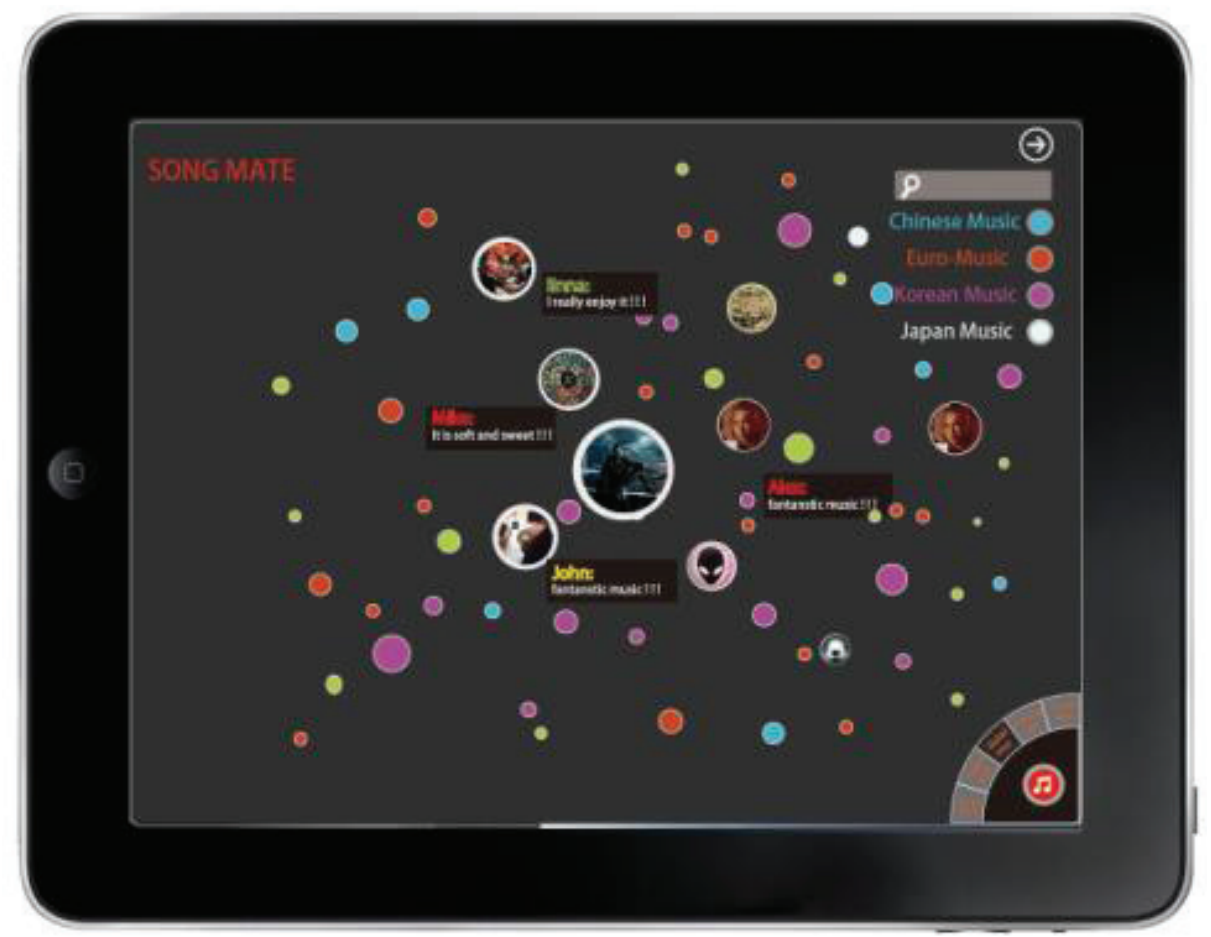

Figure 6 A group of students developed visualizations depicting relationships between users of the QQ service and their music habits. The network visualization below shows one of the ways they devised to enable users to interact with friends by means of musical affinities (used with permission of students) 

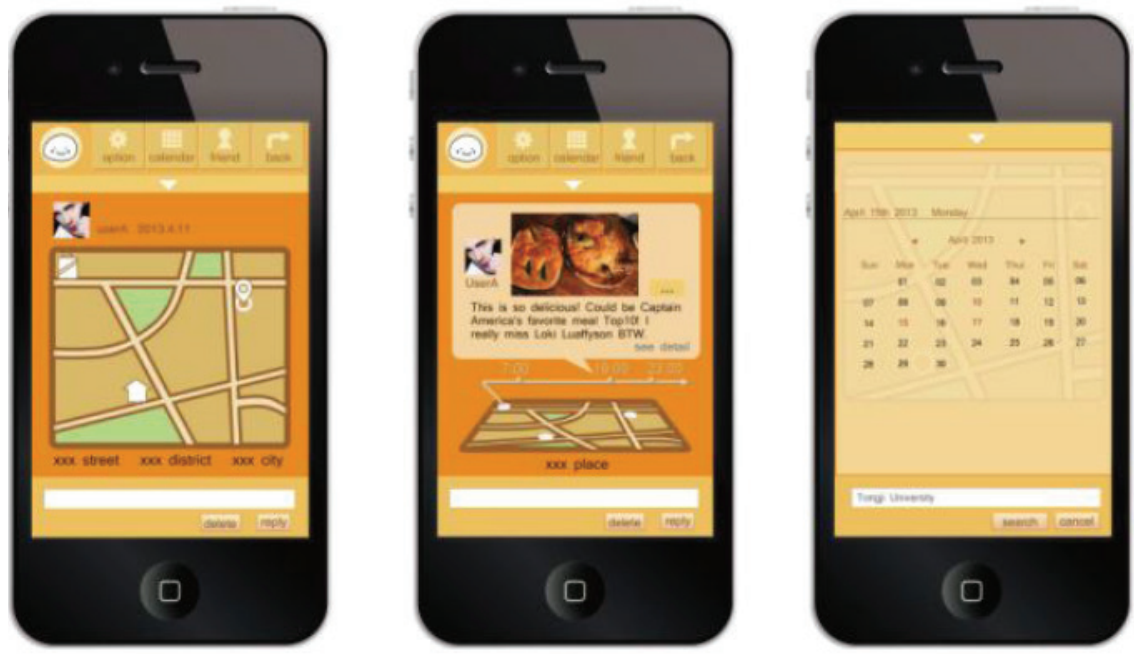

Figure 7 Another group attempted at visualizing the geo-location of QQ users over time. For that they developed ways to connect spatial and temporal data (such as timelines and calendars) with other data from the QQ application, such as messaging (used with permission of students)

The first group received further information on how to structure network visualizations, including how to encode centrality, connectedness, categories (in this case music), and quantities. The latter were presented with extra material pertaining to spatial and temporal data, such as map symbolization and strategies for encoding events over time.

Another group dealing with maps was the Dazhong Dianping group, who proposed to enable users of the service to make informed decisions about where to eat based on spatio-temporal information. Their visualization aimed at depicting the top ten results from the user's query on a map with additional information correlating how long it would take to get to a restaurant vis-a-vis how long one would have to wait to be seated (Figure 8). To help them solve this complex task, we provided them not only with information about map symbolization (as in the case of the QQ group mentioned above), but also with material on how to depict spatio-temporal data. In sum, each group's unique needs were attended individually and with additional pedagogical material that ranged from further readings to the analysis of visualizations dealing with similar problems.

Students also encountered design problems that they did not anticipate in the initial sketches, such as issues concerning the spatial constraints of smart phone screens that were not evident in the sketches. This is typical to iterative design processes, and we knew students were going to experience such issues, which we believed were best resolved at this stage and not during exploration. For example, the Altigram group decided to use color to encode different ranges of altitude. In the initial sketches for the smart phone, the 
visualization dealing with ranked images included a thin strip of colors structuring the sequence of images vertically (Figure 9). When detailing the visualization in the computer, students realized that if they kept that strip of color code they would restrict the number of images on the screen. They then removed the strip of colors, and instead designed similar functionality provided by means of interaction. We can discuss whether the removal of the code was the best decision; nonetheless, it illustrates the types of problems students faced during this development phase.

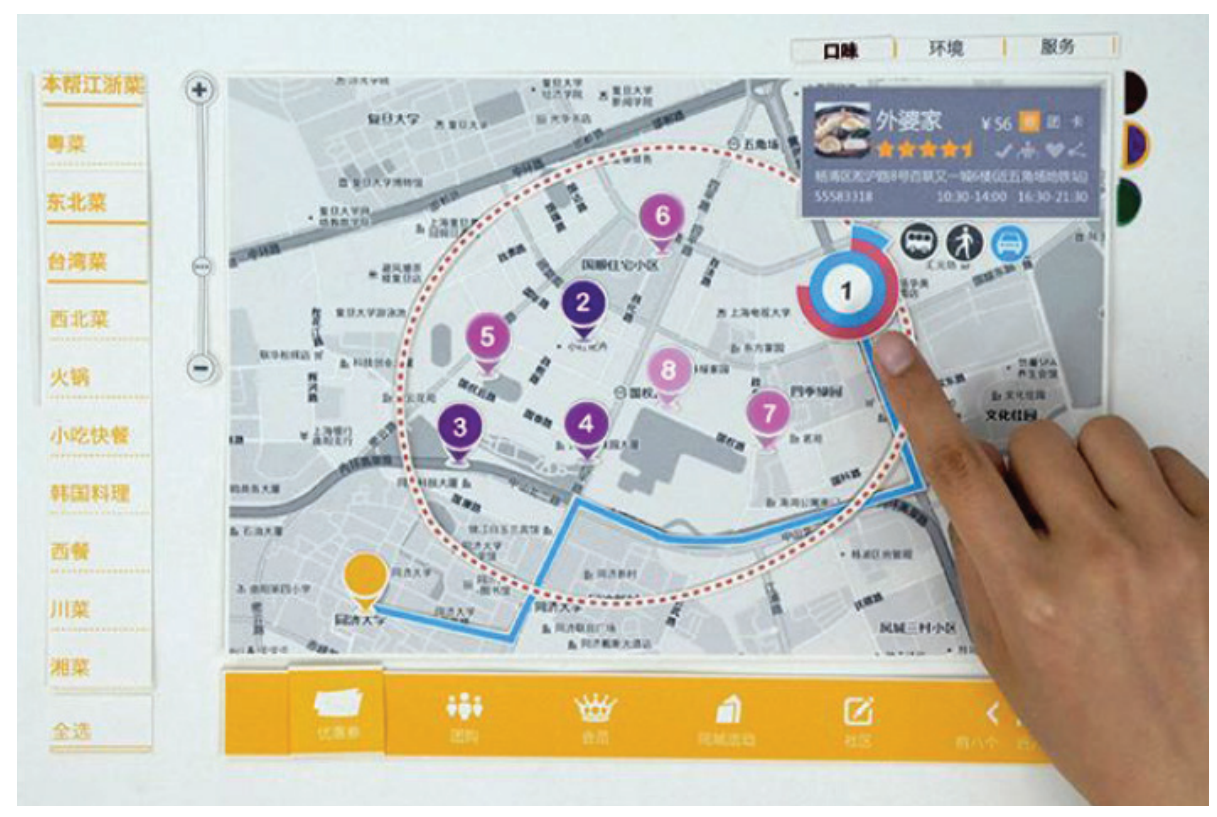

Figure 8 The map above shows the top ten results from a query at the Dazhong Dianping service and correlates each with the spatio-temporal information provided by how long it takes to get to a place as well as how long one has to wait to be seated at a table (used with permission of students)

Other complications that surfaced during the design implementation had to do with the way certain visualization features would behave. Take fore example, the WeChat group, who conceived of a visualization that would allow users to look at the accumulated number of messages exchanged overtime between friends. For that they designed a simple line graph to depict the amount of conversation across time. The concept aimed at enabling the user to interactively select a point in time and listen to the various recordings that were connected to a particular conversation (Figure 10). A great idea, but not a trivial problem to solve: how to represent a series of conversation that happen over time but might not have been necessarily contiguous or linear in time? How to consider temporal primitives of point in time and time intervals? Not to mention the constraints given by the small screen of a smart phone. 


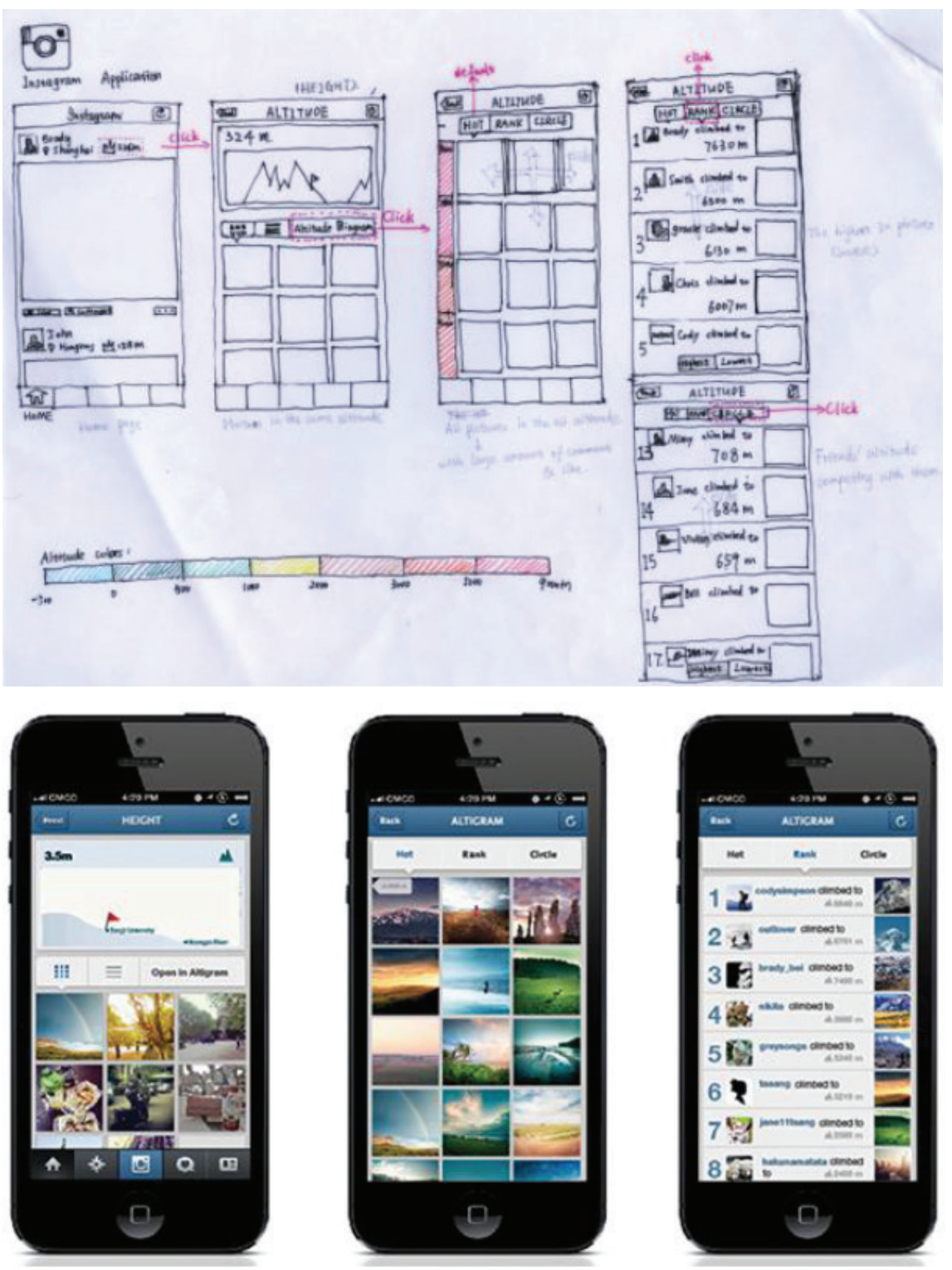

Figure 9 On the left we see the visualization sketches for Altigram app in the smart phone device. And on the right we see the detailed design of the same visualizations. Note how the color strip envisioned initially was removed in the final design (used with permission of students)

Considering that most social media applications use a combination of devices to provide their services, students had to attend to constraints imposed by the different platforms as well as the need to keep consistent encoding across devices. For example, the Dazhong Dianping group, restrained the types of information provided in the visualizations for the smart phone (Figure 11); while the Altigram group, designed different visualizations for each platform (Figure 12). The complexities brought up by designing for different devices turned out to bring interesting visualization problems. Our recommendation is that more time should be allocated to developing these issues 
further, as we see an increasing tendency in professional practice requiring the design for different platforms. We believe, students can benefit from exploring visualizations across platforms in the classroom setting.
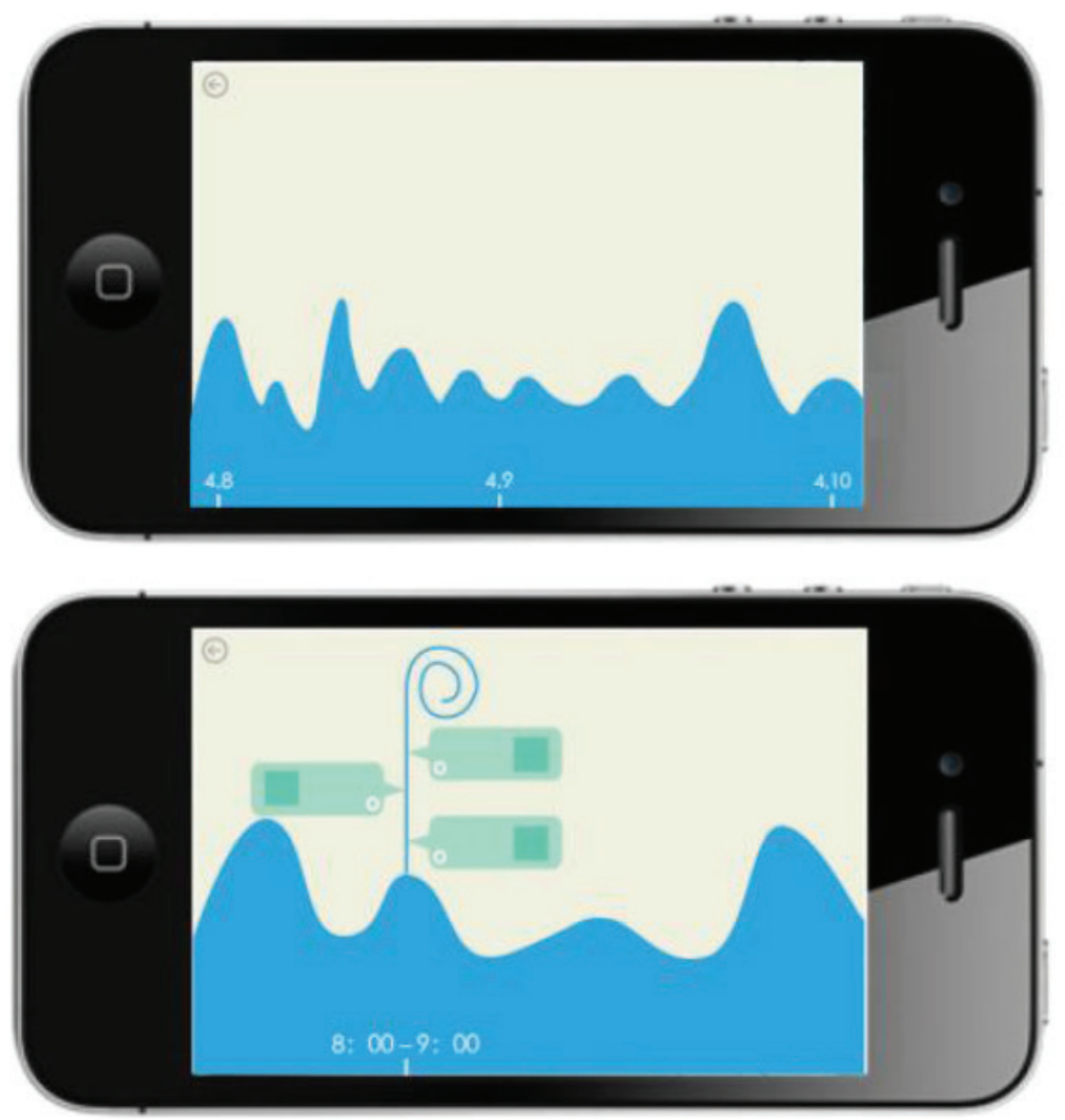

Figure 10 Two screen views of a timeline visualization of accumulated conversation between WeChat friends, where the user can select to view and interact with a particular point in time (used with permission of students)

The output of this phase shows that students devised compelling visualizations integrated into the selected social media applications, including envisioning how features would look like and behave in the chosen devices. On the other hand, we also saw that few issues remained unsolved and that they would require further development. We were aware that this would happen. Due to the short duration of the course, we did not incorporate phases that we normally would have had during the design of visualizations, such as testing and refinement. Given the introductory goal of the course, we decided to measure learning achievements by looking at the design process as a 
whole (including the outcome) and not simply by evaluating the final delivery. In other words, we measured success by examining how versed students had become in the process of designing visualizations with the appropriate structure and data encoding. For that we planned that the last phase would be devoted to developing a presentation, rather than refining the visualizations. The latter actually happened as students saw the time to refine few issues while creating the presentations, as we will see below.

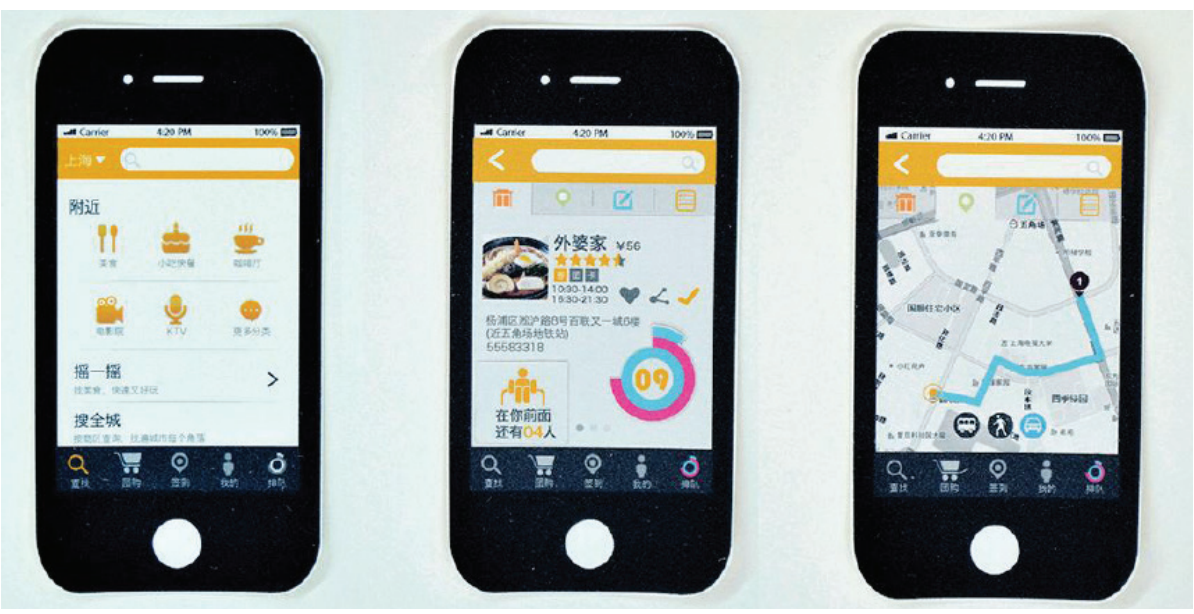

Figure 11 Few screens depicting visualization features designed for Dazhong Dianping smart phone. Compare them with Figure 8, which depicts visualization for the iPad (used with permission of students)
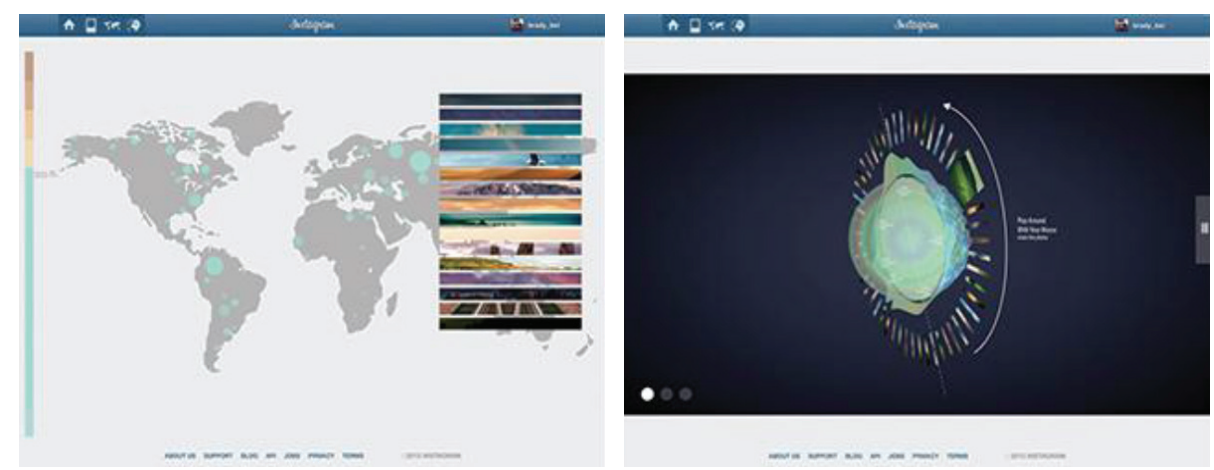

Figure 12 Two screens depicting visualization features for Altigram web site companion. Compare the differences between these visualizations with the ones devised for the smart phone in Figure 9 (used with permission of students) 


\section{Presentation}

We asked students to devise a presentation to communicate the process by which they created their solutions, as well as to demonstrate the potential and functionalities of the designed visualizations. This idea is not very different from requesting process book in studio classes. The advantage of this strategy is that students have a chance to use in their presentation many of the learned principles of communicating information in an effective manner.

In the very short period of a week, students first storyboarded and then developed presentations of their work. We encouraged students to use narrative strategies to script their presentations. They could use any means they wanted to tell their process and describe their projects. Some groups created short movies to tell their story and show how the visualization worked, while others used lecture-based presentations with the aid of projected images.

\section{Discussion}

Different from traditional information design courses in which students develop standalone projects, in this hybrid approach students had to integrate their visualizations into existing applications. Traditionally, students examine a selected topic with the objective to communicate complex issues in a clear and effective manner to a specific audience. In other words, visualization of information is examined under the lens of communication, and students devise visual displays that combine the cognitive principles of conveying meaning and facilitating discovery. In contrast, the novel method extends exploration of the potentials offered by visualization of information by including the cognitive principles of enhancing detection and recognition, providing models of actual world, and allowing manipulation of data.

Social media platforms are highly integrated into the lives of most people nowadays. By requesting students to develop new ways to engage users with these services through visualization techniques we were able to engage them into exploring and understanding the values of using visualization to deal with complex systems. For example, the first phase of the course involved conducting research to scrutinize the service beyond the students' first hand experiences with the applications. Norman argues:

The keys to coping with complexity are to be found in two aspects of understanding. First is the design of the thing itself that determines its understandability. Does it have an underlying logic, a foundation that, once mastered, makes everything fall into place? Second, is our own set of abilities and skills. Have we taken the time and effort to understand 
and master the structure? Understandability and understanding: two critical keys to mastery. (NORMAN, 2011, 4-5)

Most problems we faced related to the complexity of social media services and the short duration of the course that did not allow for certain stages in the traditional process of designing interactive visualizations, such as the refinement of the user interface and assessment of the tools by the end users. Unfortunately, the lack of refinement in the user interface design overshadowed the value of the visualizations by those unfamiliar with the process the students went through. This was evident from the comments expressed by guest critics during the final presentation, who often times pointed to user interface design issues that were on their way to understanding how the visualizations would function. In other words, the underdeveloped user interface design interfered with comprehension of the functionalities aimed by the visualizations, which were one of the objectives of the project. Our recommendation for future courses would be to extend the development phase so as to allow enough time for learning basic principles of user interface design such that they could be incorporated into the design of the final projects.

In sum, the student work, as shown here at the different stages of the process, attest to a successful achievement of our initial pedagogical goal: to ensure that students learn basic principles of structuring and encoding different types of data. We believe that this novel approach has the potential of engaging students, especially from other disciplines than graphic design, to learning the basics of visualizing information.

The current omnipresent access to data has affected research practices and has opened up new frontiers for understanding patterns in natural and social phenomena. We experience a wide use of visual techniques that, acting as cognitive devices, have been assisting researchers in several steps of their processes. We need, as a community, to share our knowledge and expertise in visualizing information and to promote visual literacy among present and future generations across disciplines. It is with this intention that we chose to share our experience with non-graphic design students at the College of Design \& Innovation (D\&I) at Tongji University, Shanghai, China.

\section{Acknowledgement}

We would like to thank students for providing us permission to reproduce the work in this paper 


\section{References}

CARD, STUART K.; MACKINLAY, JOCK; SHNEIDERMAN, BEN (Eds.) 1999. Information Visualization: Using Vision to Think. San Francisco, cA: Morgan Kaufmann. Dubberly, Hugh 2010. Creating Concept Maps. In: Concept Maps: <http://www. dubberly.com/wp-content/uploads/2010/03/ddo_creating_concept_maps. pdf>

MEIRELLES, ISABEL. 2011. Visualizing data: new pedagogical challenges. In SPINILLO, FARIAS \& PADOVANI (Eds.). Selected Readings of the 4 th Information Design International Conference: 73-83. São Paulo: SBDI, Brazilian Society of Information Design.

NORMan, D. A. 2011. Living with Complexity. Cambridge, MA: MIT Press. SIMON, H. A. 1996. The Sciences of the Artificial. Cambridge, MA: MIT Press. TVERSKY, B. 2001. Spatial Schemas in Depictions. In GATtis, M. (Ed.) Spatial Schemas and Abstract Thought: 79-112. Cambridge, MA: MIT Press.

\section{About the authors}

\section{Isabel Meirelles}

$<$ m.meirelles@neu.edu>

Art + Design Department, Northeastern University, Boston, MA, United States.

\section{Xiaohua Sun}

<xsun@tongji.edu.cn>

$\mathrm{PhD}$, College of Design \& Innovation, Tongji University, Shanghai, China. 